\title{
Are opportunities for vitamin A supplementation being utilised at primary health-care clinics in the Western Cape Province of South Africa?
}

\author{
M Hendricks $^{1, *}$, J Beardsley ${ }^{2}$, L Bourne ${ }^{3}$, B Mzamo $^{1,3}$ and B Golden ${ }^{2}$ \\ ${ }^{1}$ School of Child and Adolescent Health, University of Cape Town, 46 Sawkins Road, Rondebosch 7700, \\ Cape Town, South Africa: ${ }^{2}$ Faculty of Medicine, University of Aberdeen, Aberdeen, UK: ${ }^{3}$ Health and Development \\ Research Group, Medical Research Council, Tygerberg, South Africa
}

Submitted 11 April 2006: Accepted 16 November 2006: First published online 15 March 2007

\begin{abstract}
Objectives: To determine missed opportunities and problems relating to implementation of the Vitamin A Supplementation Programme in urban and rural regions of the Western Cape Province of South Africa.

Method: A cross-sectional survey was conducted at primary health-care (PHC) clinics in Cape Metropole, an urban region, and West Coast Winelands, a rural region, of the Western Cape. A purposive sample of clinics where more than 30 children were seen per day was drawn from 10 of the 11 districts in the Cape Metropole region and the two districts of the West Coast Winelands region. The number of children selected from each district was weighted in terms of population size for the two regions. At each clinic visited, the first five to 10 children seen on a day, and meeting the inclusion criteria for vitamin A supplementation (VAS) based on the vitamin A provincial policy guidelines, were selected. These included children with low birth weight (LBW), growth faltering, underweight and severe undernutrition, recurrent diarrhoea and lower respiratory tract infection (LRTI), tuberculosis, measles, HIV/AIDS and eye signs of vitamin A deficiency. Clinic records were reviewed following consultation with the PHC nurse to identify if the child required vitamin A, exit interviews were conducted with mothers/caregivers, and Road to Health Charts (RTHCs) were reviewed. At the end of the study, PHC managers were interviewed to determine if problems could be identified with the Programme.

Results: Forty-three of 123 (35\%) and 13 of 40 (33\%) of the fixed PHC clinics in the Cape Metropole and West Coast Winelands regions were visited, and a total of 300 children (234 from Cape Metropole, 66 from West Coast Winelands) with a mean (standard deviation) age of 24.3 (16.3) months and who met the inclusion criteria for VAS were selected. Of the total sample of children, 198 (66\%) had multiple (i.e. more than one) indication and $102(34 \%)$ had a single indication for VAS. There were a total of 617 indications for VAS in the two regions; 238 (39\%) for growth faltering, 119 (19\%) for underweight, 98 (16\%) for LBW, 70 (11\%) for LRTI, 51 (8\%) for diarrhoea, 21 (3\%) for HIV/AIDS and 20 (3\%) for tuberculosis. A total of $102(34 \%)$ of the children in the two regions received vitamin A supplements (Cape Metropole 29\%; West Coast Winelands 52\%). A record was made on the RTHC of 79 (77\%) of the children who received VAS (Cape Metropole 76\%; West Coast Winelands 79\%). Twenty-four per cent of the mothers knew why their child had been given vitamin A (Cape Metropole 29\%; West Coast Winelands 12\%). Eleven per cent of the mothers had previously heard about the Vitamin A Supplementation Programme (Cape Metropole 12\%; West Coast Winelands 6\%). More than $81 \%$ of PHC managers indicated that health staff had been trained to implement the Vitamin A Supplementation Programme. The main problems identified by health staff in the two regions were lack of vitamin A capsules, inadequate training and difficulties in implementing the Programme.

Conclusions: Opportunities to administer vitamin A were underutilised in both regions. Recommendations such as improving mothers' awareness of the benefits of vitamin A and training of PHC nurses were made to the provincial Department of Health and are being implemented to improve the effectiveness of the Programme.
\end{abstract}

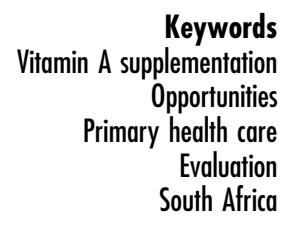


In 1994, a national survey showed that 33\% of South African children aged $<6$ years had marginal vitamin A status (i.e. serum retinol level below $\left.20 \mu \mathrm{g} \mathrm{dl}^{-1}\right)^{1}$. Children in rural areas and whose mothers were poorly educated had the highest prevalence of vitamin A deficiency (VAD) ${ }^{1}$. Based on these findings, a range of interrelated interventions to eliminate VAD were recommended by the South African Vitamin A Consultative Group ${ }^{1}$. These included vitamin A supplementation (VAS) for postpartum women and children aged $<6$ years in the short term; food fortification and dietary diversification, aimed at improving the consumption of vitamin-A-rich foods, in the medium to long term; and public health measures such as the promotion of breast-feeding, improved immunisation coverage (particularly against measles), nutrition-related management of disease and overall improvements in socio-economic status.

VAS was found to reduce all-cause mortality in children aged between 6 months and 5 years by $23 \%{ }^{2}$, measles mortality by $50 \%{ }^{3}$ and mortality from HIV by $63 \%{ }^{4}$. For tuberculosis, vitamin A together with zinc was found to lead to more rapid elimination of tubercle bacilli from sputum samples 5 .

The South African national Department of Health endorsed the implementation of a national Vitamin A Supplementation Programme as part of a broader strategy to eliminate VAD and reduce morbidity and mortality in the under-6s. A study looking at the feasibility of implementing such a programme in South Africa showed that this could be a cost-effective intervention for eliminating the problem of $\mathrm{VAD}^{6}$. The Nutrition and Maternal, Child and Women's Health (MCWH) Subdirectorates in the Western Cape Province of South Africa established a medically targeted Vitamin A Supplementation Programme in 1999 for children at risk of VAD based on provincial case management guidelines, which were developed to guide implementation of the Programme. The main reasons for developing a targeted distribution programme were: (1) the relatively high cost of vitamin A capsules at the time, following the award of a tender to the pharmaceutical company that was then the sole manufacturer of vitamin A capsules in the country, and (2) because of delays in implementing the national programme; the Vitamin A Supplementation Programme in the Western Cape was implemented 2 years prior to the national programme (Provincial Government of the Western Cape, personal communication, 2006). Medical targeting has been reported as a cost-effective way of getting vitamin A to a subpopulation of children who need it. It is also seen as feasible because sick children are brought to the health facility, thereby facilitating distribution of vitamin A supplements ${ }^{7}$. Provincial guidelines for VAS were developed by a provincial reference group, which included representatives from the provincial Department of Health, service organisations and the three universities in the province ${ }^{8}$. A decision was taken to include children at risk of VAD as specified in the guidelines of the World Health Organization (WHO) ${ }^{9}$ as well as other high-risk groups, e.g. children with HIV/ AIDS and tuberculosis. The VAS guidelines targeted highrisk mothers (within 4 weeks of delivery) and children with low birth weight (LBW), underweight, growth faltering and severe malnutrition, recurrent diarrhoea and lower respiratory tract infection (LRTI), measles, tuberculosis, HIV/AIDS and those with eye signs of VAD ${ }^{8}$. The guidelines were implemented at primary health-care (PHC) facilities that provide comprehensive child health care, maternity obstetric units and hospitals.

A formal evaluation of the implementation of the Vitamin A Supplementation Programme has until now not been performed in the province, although there are anecdotal reports relating to poor coverage in some areas. Thus the objectives of the present study were to determine missed opportunities and problems relating to implementation of the Vitamin A Supplementation Programme at PHC facilities in urban and rural regions of the Western Cape Province of South Africa. Based on the findings, recommendations would be made to strengthen the Vitamin A Supplementation Programme in the province.

\section{Method}

\section{Study area}

The study was undertaken in Cape Metropole, an urban region, and West Coast Winelands, a rural region, situated in the Western Cape Province of South Africa. The total population in Cape Metropole in 2001 was 2.9 million, making up $65 \%$ of the provincial population ${ }^{10}$. The population of under- 5 s was estimated to be 259572 . The infant mortality rate was 25 per 1000 live births and lower than the national average of 45 per 1000 live births ${ }^{10}$. The main causes of infant mortality were HIV/AIDS, diarrhoeal disease, LBW and pneumonia; Cape Metropole had one of the highest incidences of tuberculosis in the country, estimated to be 638 per 100000 in $2002^{10}$. The total population in 2001 for the West Coast Winelands region was 282672 and the population of under-5s numbered $56249^{10}$. Currently there are no data on the infant mortality rate for this region of the province. Most families lived on farms and the adults were employed in the agricultural and fishing industries.

\section{Study design and sampling}

A cross-sectional study was carried out at PHC clinics in the two regions of the province. The study was undertaken between 1 April 2003 and 31 October 2004. Based on the maximum VAS coverage rate of $30 \%$ found in previous studies (Department of Health, personal communication, 2003), an estimate of the coverage in this study population, with an absolute precision of 5\% at the $95 \%$ level of confidence, required a sample size of about 
323 children ${ }^{11}$. The study aimed to include children from the 11 districts in Cape Metropole region and the two districts in West Coast Winelands region. Each of the two regions was stratified by district and the selection of children per district was weighted in terms of population size (Tables 1 and 2). The study covered 123 and 40 fixed PHC clinics in Cape Metropole and West Coast Winelands region, respectively. A purposive sample of PHC clinics was drawn from each district where more than 30 children were seen a day and where comprehensive child health care was provided on a daily basis. This criterion was met by 89 (55\%) of the PHC clinics (63 in Cape Metropole; 26 in West Coast Winelands). The reason for selecting the larger or busier PHC clinics was to determine how well the Vitamin A Supplementation Programme was being implemented at clinics where there is often a high curative workload. The study aimed to cover $40 \%$ of all clinics in the Cape Metropole and West Coast Winelands regions as it was estimated that this proportion of clinics would ensure the intended sample size would be reached. The first five to 10 children who required VAS in terms of the provincial case management guidelines ${ }^{8}$ were recruited on the day of the study. These included children aged 6 months to 6 years of age, not given vitamin $\mathrm{A}$ in the previous 6 months, with one or more of the following conditions: LBW $(<2500 \mathrm{~g})$; more than one episode of diarrhoea or LRTI in the previous 3 months; HIV/AIDS, tuberculosis or measles; eye signs of vitamin A deficiency; and growth faltering, underweight or severe malnutrition. Children with vomiting, those who had received vitamin A in the previous 6 months and where the mother did not give consent were excluded from the study.

\section{Data collection}

All of the data collection at clinics was done by a dietitian, a registered nurse, a fifth-year medical student and a fieldworker. Clinic records were reviewed following consultation with the PHC nurse to identify the child's underlying problem and to assess if the child required

Table 1 Number (\%) of children selected by district in Cape Metropole

\begin{tabular}{lcrrrr}
\hline & \multicolumn{2}{c}{$\begin{array}{c}\text { Population } \\
<5 \text { years }\end{array}$} & & \multicolumn{2}{c}{$\begin{array}{c}\text { Children } \\
\text { selected }\end{array}$} \\
\cline { 2 - 3 } \cline { 5 - 6 } District & $n$ & $\%$ & & $n$ & $\%$ \\
\hline Athlone & 28696 & 11 & & 19 & 8 \\
Blaauwberg & 12308 & 5 & & 17 & 7 \\
Central & 16527 & 6 & & 0 \\
Helderberg & 11473 & 4 & 16 & 7 \\
Khayelitsha & 32391 & 12 & 41 & 18 \\
Mitchell's Plain & 26309 & 10 & 31 & 13 \\
Nyanga & 16282 & 6 & 26 & 11 \\
Oostenberg & 29206 & 11 & 18 & 8 \\
South Peninsula & 35524 & 14 & 29 & 12 \\
Tygerberg East & 19693 & 8 & 11 & 5 \\
Tygerberg West & 31163 & 12 & 26 & 11 \\
Total & 259572 & 100 & 234 & 100 \\
\hline
\end{tabular}

Table 2 Number (\%) of children selected by district in West Coast Winelands

\begin{tabular}{lccccr}
\hline & \multicolumn{2}{c}{$\begin{array}{c}\text { Population } \\
<5 \text { years }\end{array}$} & & \multicolumn{2}{c}{$\begin{array}{c}\text { Children } \\
\text { selected }\end{array}$} \\
\cline { 2 - 3 } \cline { 5 - 6 } District & $n$ & & & & \\
\cline { 2 - 3 } & 28241 & 51 & & 40 & 61 \\
Boland & 28008 & 49 & & 26 & 39 \\
West Coast & 56249 & 100 & & 66 & 100 \\
Total & & & & \\
\hline
\end{tabular}

vitamin A. In the case where the child fulfilled the inclusion criteria (i.e. based on the provincial case management guidelines) for VAS, the mothers/caregivers of recruited subjects were given a structured exit interview using a questionnaire which aimed to identify: whether supplementation was indicated at the time and, if so, whether the PHC nurse identified that fact and gave the child vitamin A; and whether the mother knew why the child had been given vitamin A and knew about the Vitamin A Supplementation Programme. The weight of the child was recorded on the local clinic scale (Masskot, Medway or UC-321 scale) to the nearest $0.1 \mathrm{~kg}$. The child's Road to Health Chart (RTHC) was reviewed at the same time to assess whether vitamin A was recorded when given to the child as recommended in the provincial policy guidelines. If a child had an indication for VAS and was not contraindicated, it was noted that an opportunity had been missed.

The PHC nurses working in the selected clinics were unaware that the study focused specifically on VAS. After data had been collected, time was taken to interview the PHC nursing managers to get their perspectives on the main problems relating to implementing the Vitamin A Supplementation Programme.

\section{Data analysis}

Summary statistics (percentages, means and standard deviations, (SD)) were determined for descriptive variables. In terms of anthropometry, underweight and stunting were expressed as the proportion of individuals with $Z$-score $<-2$ SD below the mean values of the National Center for Health Statistics/WHO reference population $^{12}$.

\section{Etbical issues}

Permission for the study was granted by the Research Ethics Committee of the University of Cape Town. The major ethical issue with this study was ensuring that the child's health remained paramount. In situations where a missed opportunity was identified it was necessary to arrange rectification via a follow-up clinic appointment. Names of children who did not receive vitamin A were provided to the PHC clinics so that follow-up appointments could be arranged. Consent was obtained from the mothers/caregivers at the time of the exit interview for participation in the study. PHC workers were assured 
of the anonymity of the findings to remove any concerns about being judged.

\section{Results}

We visited 43 (35\%) and 13 (33\%) of the fixed PHC clinics in the Cape Metropole and West Coast Winelands regions, respectively (Table 3). The total sample included 234 and 66 children who met the inclusion criteria for VAS from the two respective regions. We selected children from each of the districts in Cape Metropole with the exception of Central District (Table 1), where there were few clinic attendees and no children could be identified for inclusion in the study; we were also requested not to use the largest clinic in this district as another research project was being conducted there at the time (Department of Health, personal communication, 2003). Most of the children were selected from the larger districts such as Khayelitsha (18\%), Mitchell's Plain (13\%), South Peninsula (12\%) and Tygerberg West (11\%). In the West Coast Winelands region, we selected children from both districts: 40 (61\%) children from Boland District (Stellenbosch and Drakenstein Sub-districts) and 26 (39\%) children from West Coast District (Saldanha and Swartland Sub-districts) (Table 2). Fewer children were selected from the latter district because of difficult access and long distances that had to be travelled in reaching the clinics.

The total sample included 140 (47\%) boys and 160 (53\%) girls, with a similar percentage of boys and girls from the two regions (Table 3 ). The mean (SD) age of the children was 24.3 (16.3) months and their mean birth weight was 2788 (627) g. The weight-for-age $Z$-score was $<-2$ SD in 116 (39\%) of the children.

Among the 234 children in Cape Metropole there were 463 indications for VAS (Table 4), which included growth faltering ( $n=200,43 \%)$, underweight ( $n=80,17 \%)$, LBW $(n=59,13 \%)$, recurrent LRTI $(n=51,11 \%)$, recurrent diarrhoea $(n=43,9 \%)$, HIV/AIDS $(n=17,4 \%)$ and tuberculosis ( $n=13,3 \%$ ). Of the children, 148 (63\%) had multiple indications (i.e. more than one indication), while $86(37 \%)$ had a single indication for VAS. Among the 66
Table 4 Indications for vitamin A supplementation in Cape Metropole and West Coast Winelands regions

\begin{tabular}{|c|c|c|c|c|c|c|}
\hline \multirow[b]{2}{*}{ Indication } & \multicolumn{2}{|c|}{$\begin{array}{c}\text { Cape } \\
\text { Metropole }\end{array}$} & \multicolumn{2}{|c|}{$\begin{array}{l}\text { West Coast } \\
\text { Winelands }\end{array}$} & \multicolumn{2}{|c|}{ Total } \\
\hline & $n$ & $\%$ & $n$ & $\%$ & $n$ & $\%$ \\
\hline Growth faltering & 200 & 43 & 38 & 25 & 238 & 39 \\
\hline Underweight & 80 & 17 & 39 & 25 & 119 & 19 \\
\hline LBW & 59 & 13 & 39 & 25 & 98 & 16 \\
\hline LRTI & 51 & 11 & 19 & 12 & 70 & 11 \\
\hline Diarrhoea & 43 & 9 & 8 & 5 & 51 & 8 \\
\hline HIV/AIDS & 17 & 4 & 4 & 3 & 21 & 3 \\
\hline Tuberculosis & 13 & 3 & 7 & 5 & 20 & 3 \\
\hline Total & 463 & 100 & 154 & 100 & 617 & 100 \\
\hline
\end{tabular}

LBW - low birth weight; LRTI - lower respiratory tract infection.

children in the West Coast Winelands region there were 154 indications for VAS, which included underweight $(n=39,25 \%)$, LBW $(n=39,25 \%)$, growth faltering ( $n=38,25 \%)$, recurrent LRTI $(n=19,12 \%)$, recurrent diarrhoea $(n=8,5 \%)$, tuberculosis $(n=7,5 \%)$ and HIV/ AIDS $(n=4,3 \%)$. Multiple indications were found in 50 (76\%) children, while 16 (24\%) children had a single indication for VAS.

Figure 1 outlines the practices of PHC nurses regarding VAS. Of the children in the two regions, 102 (34\%) were given vitamin A supplements (Cape Metropole: $n=68$, 29\%; West Coast Winelands: $n=34,52 \%$ ). In the Cape Metropole region VAS coverage rates ranged from $7 \%$ (South Peninsula District) to 67\% (Oostenberg District); in the West Coast Winelands region it ranged from 53\% (Boland District) to 59\% (West Coast District). Lack of administration of vitamin A was not linked to unavailability of vitamin A capsules at any of the clinics; often in the same clinic there were children who were given vitamin A while opportunities were missed in supplementing those in whom vitamin A was indicated. Of the children who received VAS, a record was made on the RTHC of 79 (77\%) (Cape Metropole: $n=52,76 \%$; West Coast Winelands: $n=27,79 \%)$. Twenty-four (24\%) mothers (Cape Metropole: $n=20,29 \%$; West Coast Winelands: $n=4,12 \%$ ) in the two regions knew why

Table 3 Sociodemographic and anthropometric status of children

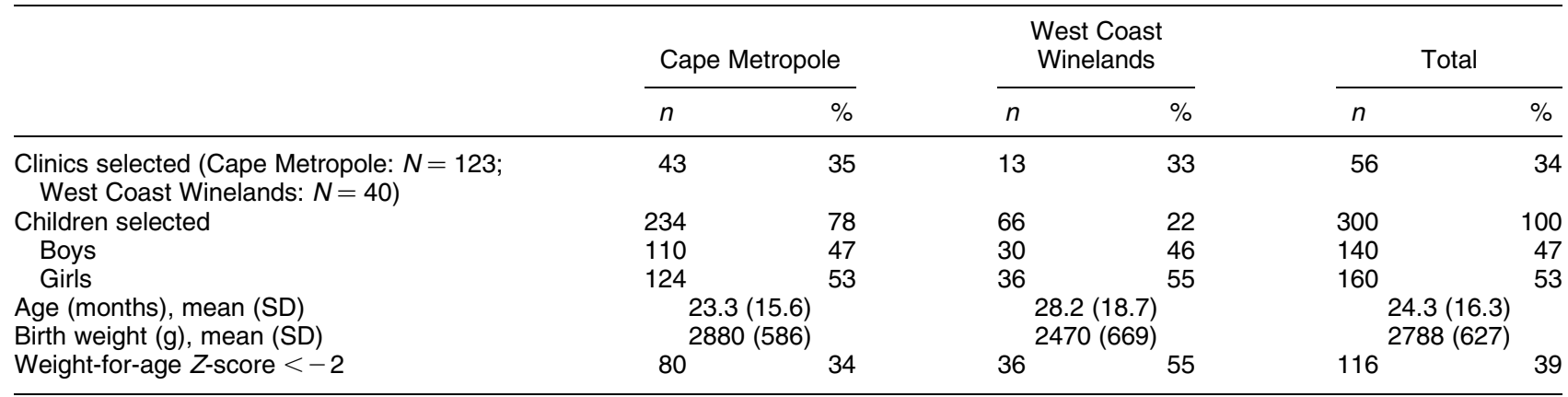

SD - standard deviation. 


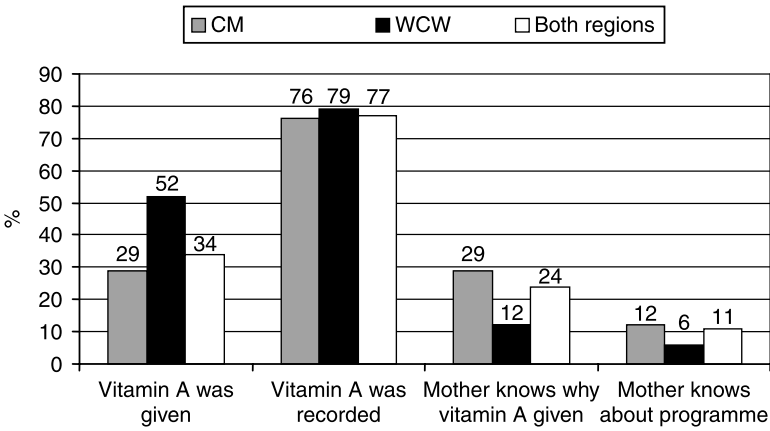

Fig. 1 Practices of primary health-care nurses regarding vitamin A supplementation (CM - Cape Metropole; WCW - West Coast Winelands; RTHC - Road to Health Chart)

their child had been given vitamin A supplements; 33 (11\%) mothers (Cape Metropole: $n=29$, 12\%; West Coast Winelands: $n=4,6 \%$ ) had heard about the Vitamin A Supplementation Programme.

Forty-three (77\%) PHC nursing managers (Cape Metropole: $n=31,72 \%$; West Coast Winelands: $n=12,92 \%$ ) based at the different clinics were available for an interview at the end of the study. Of these, 35 (81\%) indicated that the PHC nursing staff had received training on VAS. Of the PHC nursing managers, 37 (86\%) identified problems while six (14\%) did not identify problems with the Vitamin A Supplementation Programme. Problems identified in the two regions included difficulties in implementing the Programme by 16 (43\%), which included staff forgetting to check the vitamin A protocol and to record the vitamin A given, and confusion over the indications for VAS; running out of vitamin A capsules at times by 13 (35\%); and inadequate training by eight (22\%).

Recommendations made by PHC nursing managers in the two regions included: increasing maternal/caregiver awareness of VAS $(n=14,32 \%)$; improvements in the intensity and frequency of training $(n=12,28 \%)$; ensuring adequate supplies of capsules $(n=2,5 \%)$; targeting crèches and pre-schools $(n=2,5 \%)$; training other categories of health personnel $(n=2,5 \%)$; increasing staff awareness $(n=2,5 \%)$; and reminding staff to record vitamin A on the RTHC $(n=2,5 \%)$. Seven (16\%) of the managers made no recommendations for improving the Programme.

\section{Discussion}

The rationale for evaluating the Vitamin A Supplementation Programme among urban and rural children in the Western Cape was for comparative purposes. PHC clinics in urban areas are better-resourced and staff have easier access to training and support for programme implementation; on the other hand, rural clinics are less wellresourced, staff have less access to training and there is often less support for implementation of programmes. Compared with urban children, rural children are worse off in terms of socio-economic status, access to health services and have been found to have higher prevalences of undernutrition and micronutrient deficiency ${ }^{1,13}$. Given these differences it was important to assess the Vitamin A Supplementation Programme in rural and urban regions, to determine what input and support would be needed, and whether these would differ depending on the context.

The findings of the study showed that uptake of VAS was higher among rural than urban children (52\% vs. 29\%). Despite this, these coverage rates are low considering that these were children at high risk of VAD and were from communities in the Western Cape where VAD is a significant public health problem. By comparison, coverage rates reported nationally for children aged 12-59 months in the other eight provinces, where universal VAS has been implemented ${ }^{14}$, are lower than that reported for the two regions in the Western Cape (13\% vs. 34\%). In another study that focused on opportunities for VAS among village children in Malawi, the coverage rate ranged from 2.4 to $24.5 \%$ and several missed opportunities were reported ${ }^{15}$. However, good coverage rates have been achieved in other programmes. For example, in a study conducted in Pakistan, VAS coverage was found to be $74.8 \%{ }^{16}$. Similarly in Ghana, vitamin A coverage was estimated to be $80 \%$ and was associated with a significant decline in child mortality and morbidity from measles and diarrhoea $^{17}$. In these studies undertaken in Malawi, Pakistan and Ghana vitamin A capsules were delivered alongside immunisations (i.e. universal supplementation) and, in the case of Ghana, it also included periodic supplementation of children aged $>6$ months as well as postpartum mothers. Our study differed from these in that it was a medically targeted vitamin distribution programme and evaluations of similar programmes have not been previously reported.

There was considerable variation in VAS coverage rates across the different health districts in Cape Metropole, although this was less marked for the West Coast Winelands region. The reason for the wide variation in vitamin $\mathrm{A}$ coverage needs to be explored further, as training in VAS was standardised across the various districts in Cape Metropole. Good coverage of VAS is dependent on effectively trained and committed health workers who can communicate effectively with consumers ${ }^{7}$. The low coverage rates in this study could indicate a lack of motivation of staff who are required to manage a high curative workload and who may not recognise VAS as an important health-promoting and child-survival strategy ${ }^{18}$. Similarly in the study conducted in Malawi, the authors attributed the poor vitamin A coverage rates to lack of knowledge and motivation of health staff ${ }^{11}$. Whatever the cause, failure to provide vitamin A to children who need it is likely to have a significant impact on morbidity and mortality, and is an issue that must be addressed.

The main indications for VAS in both rural and urban children included growth faltering, underweight and LBW. 
In both settings children had multiple rather than single indications for VAS. The presence of these multiple indications indicates the link between undernutrition and recurrent infections: infections are known to deplete the body's stores of vitamin A. This kind of vicious cycle makes a strong case for VAS and emphasises how much VAS is needed by the children from these communities. However, some of the indications for VAS appeared less frequently than might have been expected. For example, tuberculosis is highly prevalent in the Western Cape and this indication for VAS could be deceptively low in the present study because these children attended a special clinic and seldom had their RTHC with them.

In both rural and urban areas, few mothers were aware of the Vitamin A Supplementation Programme and the benefits of vitamin A for their children. Barriers to effective communication with mothers and promotion of the Programme may have included differences in language and educational achievement, which could be difficult for a health worker to address when working in an overcrowded health facility. However, health promotion is integral to programmes such as the Integrated Management of Childhood Illness (IMCI) strategy, which is currently implemented at PHC clinics in the province; it is also vital to the success of the Vitamin A Supplementation Programme. Community awareness through social marketing of the benefits vitamin A supplements together with health promotion among consumers could create a demand for vitamin A capsules, which, in turn, would increase awareness amongst the health staff. This kind of positive feedback would ensure that VAS is not overlooked ${ }^{7}$.

To avoid multiple dosing, it is important to record vitamin A administration on the child's RTHC ${ }^{19}$. The rates of recording administered doses of vitamin A on children's RTHCs was $67 \%$ and $79 \%$ for the urban and rural regions, respectively, and could be addressed through training as this is one of the few records indicating that the child received vitamin $\mathrm{A}$.

Staff in both rural and urban sites felt that training was inadequate and that it did not equip them adequately to implement the Programme. This appeared to be more prevalent in the rural sites and could be related to the fact that the IMCI strategy had not yet been rolled out there at the time of the study. Some health workers interviewed, particularly in the Cape Metropole region, indicated that they had experienced problems with the supply of vitamin A capsules. These are factors that could have undermined the effectiveness of the Programme.

The main limitations of this study are the use of purposive sampling of larger clinics and the exclusion of smaller clinics where VAS coverage may have been better. However, it was important to assess practices at larger clinics, which make up the bulk of the PHC services and where most children are seen. As clinics were included from virtually all districts in the two regions, it is likely that this was a representative sample of provincial PHC clinics and that the findings are an accurate reflection of the practices of health staff regarding VAS.

Based on the results of the study, it was recommended that: the Nutrition Sub-directorate establish contact with all the agencies responsible for implementing the Vitamin A Supplementation Programme, including the MCWH Subdirectorate, health promotion, human resources and pharmaceutical services; the training of health workers be reviewed and made consistent with strategies such as the IMCI; and advocacy and communication be promoted about the importance of vitamin A for health and child survival at all levels, from consumers to policy-makers, to improve coverage. Since undertaking this study, several of these recommendations have been implemented, and the Vitamin A Supplementation Programme in the Western Cape has moved to universal supplementation of preschool children attending health facilities. Improved coverage rates are already being seen in some of the districts in the province (Department of Health, personal communication, November 2006). However, this study emphasises that it is essential that health workers are trained to assess the VAS status of children at every clinic visit to avoid missed opportunities. Regular monitoring of vitamin A coverage rates in the province must be undertaken and problems in the delivery of the Programme should be identified and addressed. Vitamin A capsules given must be recorded on the RTHC and this practice needs to be regularly assessed during supervisory visits to health facilities.

\section{Conclusion}

Opportunities to administer vitamin A were missed in several districts of the Cape Metropole and the West Coast Winelands regions during the period of the study. The main problems in implementing VAS were related to suboptimal delivery of vitamin A capsules to the children who needed them, inadequate promotion and lack of awareness of the Vitamin A Supplementation Programme by mothers/caregivers, and inadequate training of health workers. The provincial Department of Health has addressed several of these issues, but ongoing monitoring and action are needed to improve the effectiveness of the Programme.

\section{Acknowledgements}

Sources of funding: The project was funded by the Nutrition Sub-directorate of the Provincial Government of the Western Cape, the Medical Research Council and the School of Child and Adolescent Health of the University of Cape Town.

Conflict of interest declaration: None.

Authorship responsibilities: M.K.H. - study conception and design, data analysis and interpretation, drafting of 
manuscript, grant writing, facilitation of performance and supervision of study; J.B. - study conception and design, data collection, analysis and interpretation, drafting of manuscript, data management; L.B. - study design, drafting and revision of manuscript, funding, administrative and technical support; B.M. - data acquisition, drafting of manuscript, administrative and technical support and supervised performance of study; B.G. study design, supervision of writing and administrative support.

Acknowledgements: We would like to acknowledge Hilary Goeiman and Luzette van Niekerk of the Nutrition Sub-directorate in the Western Cape for supporting the study; the mothers and health staff at clinics in the Cape Metropole and West Coast Winelands regions for participating in the study; and the funding organisations.

\section{References}

1 South African Vitamin A Consultative Group (SAVACG). Children aged 6-71 months in South Africa, 1994; Their Anthropometric, Vitamin A, Iron and Immunisation Coverage Status. Isando, Gauteng: SAVACG, 1995.

2 Beaton GH, Martorell R, Aronson KJ, Edmonston B, McCabe $\mathrm{G}$, Ross AC, et al. Effectiveness of Vitamin A Supplementation in the Control of Young Child Morbidity and Mortality in Developing Countries. Administrative Committee on Coordination/Sub-committee on Nutrition State-of-the-Art Series, Nutrition Policy Discussion Paper No. 13. Toronto: International Nutrition Program, University of Toronto, 1993.

3 Bloem M, de Pee S, Darnton-Hill I. New issues in developing effective approaches for the prevention and control of vitamin A deficiency. Food and Nutrition Bulletin 1998; 19(2): 137-48.

4 Fawzi WW, Roger L, Hertzmark E, Fataki MR, Herrera MG, Ndossi G, Spiegelman D. A randomized trial of vitamin A supplements in relation to mortality among human immunodeficiency virus-infected and uninfected children in Tanzania. Pediatric Infectious Disease Journal 1999; 18(2): $127-33$.

5 Karyadi E, West CE, Schultink W, Nelwan RH, Gross R, Amin $\mathrm{Z}$, et al. A double blind, placebo-controlled study of vitamin $A$ and zinc supplementation in persons with tuberculosis in Indonesia: effects on clinical response and nutritional status. American Journal of Clinical Nutrition 2002; 75(4): 720-7.

6 Saitowitz R, Hendricks M, Fiedler J, le Roux I, Hussey G, Makan B. A proposed vitamin A supplementation programme for South Africa - design, coverage and cost. South African Medical Journal 2001; 91(9): 755-60.
7 World Bank. Enriching Lives: Overcoming Vitamin and Mineral Malnutrition in Developing Countries. Washington, DC: International Bank for Reconstruction and Development/World Bank, 1994

8 Provincial Administration of the Western Cape (PAWC), Maternal, Child and Women's Health and Nutrition Subdirectorates, Provincial Reference Group. Integrated Case Management Guidelines: Vitamin A Supplementation. Cape Town, Western Cape: Department of Health and Social Services, PAWC, 1999.

9 World Health Organization (WHO)/United Nations Children's Fund/International Vitamin A Consultative Group Task Force. Vitamin A Supplements: A Guide to their Use in the Treatment and Prevention of Vitamin A Deficiency and Xerophthalmia. Geneva: WHO, 1997.

10 Department of Health of the Western Cape. Community Health Services Organisation Cape Town: Annual Report, April 2002-March 2003 (unpublished).

11 Vaughan JP, Morrow RH, eds. Manual of Epidemiology for District Health Management. Geneva: World Health Organization, 1993

12 Hamill PV, Drizd TA, Johnson CL, Reed RB, Roche AF, Moore WM. Physical growth: National Center for Health Statistics percentiles. American Journal of Clinical Nutrition 1979; 32(3): 607-29.

13 Labadarios D, Steyn NP, Maunder E, MacIntyre U, Swart R, Gericke G, et al. The National Food Consumption Survey (NFCS): Children aged 1-9 years, South Africa, 1999. Pretoria, Gauteng: Department of Health, 2000.

14 Department of Health (DOH)/United States Agency for International Development (USAID). Vitamin A Supplementation: Training Curriculum. Pretoria: DOH/USAID, 2005.

15 Berger RA, Courtright P, Barrows J. Vitamin A capsule supplementation in Malawi villages: missed opportunities and possible interventions. American Journal of Public Health 1995; 85(5): 718-9.

16 Bharmal FY, Omair A. Evaluation of vitamin A supplementation in Gulshan-e-Sikandarabad. Journal of the Pakistan Medical Association 2001; 51(7): 247-50.

17 David P. Evaluating the Vitamin A Programme in Northern Ghana: Has it Contributed to Improved Child Survival? Boston, MA: John Snow Inc., 2003.

18 Dalmiya N, Palmer A, Darnton-Hill I. Sustaining vitamin A supplementation requires a new vision. Lancet 2006; 368(9541): 1052-4.

19 World Health Organization (WHO)/United Nations Children's Fund (UNICEF). Expanded Programme on Immunization. Integration of Vitamin A Supplementation with Immunization: Policy and Programme Implication. Report of a Meeting, UNICEF, New York, 12-13 January 1998. Geneva: WHO, 1998. 